\title{
Facile Synthesis of Unsymmetrical 1,1-Diaryl-2,2-difluoroethenes via Stepwise Coupling of 1,1-Dibromo-2,2-difluoroethenes
}

Takeshi Fujita, Naoto Suzuki, Tomohiro Ichitsuka, and Junji Ichikawa*

Division of Chemistry, Faculty of Pure and Applied Sciences, University of Tsukuba, Tsukuba, Ibaraki 305-8571, Japan

*Corresponding author. Fax: +81-29-853-4237

E-mail address: junji@chem.tsukuba.ac.jp (J. Ichikawa).

\begin{abstract}
Unsymmetrical 1,1-diaryl-2,2-difluoroethenes were synthesized from 1,1-dibromo-2,2-difluoroethene, which is commercially available, via the Suzuki-Miyaura coupling in a stepwise fashion. Suitable ligands for each coupling process were used to achieve selective synthesis of these diaryldifluoroethenes.
\end{abstract}

Keywords: Difluoroalkene, Diarylethene, Suzuki-Miyaura coupling, Dibromoethene, Arylboronic acid, Palladium, Ligand

\section{Introduction}

1,1-Diaryl-2,2-difluoroethenes are an important class of gem-difluoroalkenes, which have the potential to be used for a wide range of purposes, including functional materials and medicines such as photoreceptors [1], anticancer drugs [2], and dermatological agents [3]. These compounds are also expected to be bioisosteres of biologically active diarylketones [4]. Despite their versatility, the supply of 1,1-diaryl-2,2-difluoroethenes has been limited because of difficulties involved in synthesizing them (Scheme 1) [5,6]. Conventional synthetic methods, especially for unsymmetrically disubstituted 1,1-diaryl-2,2-difluoroethenes, have mostly required difluorovinylidene species to be synthesized as key intermediates, bearing both a metal functional group and a halogen (or pseudohalogen) substituent at the carbon $\beta$ to the fluorine substituents [6]. This is due to selective introduction of two different aryl groups. The preparation of such intermediates is troublesome and requires extra synthetic steps. 


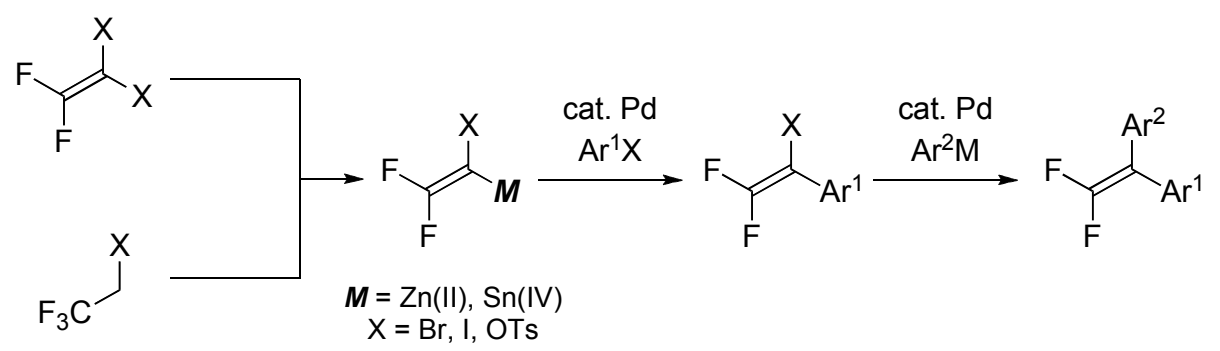

Scheme 1. Conventional synthesis of 1,1-diaryl-2,2-difluoroethenes.

A straightforward synthesis of unsymmetrical 1,1-diaryl-2,2-difluoroethenes might be achieved simply with two reactivity-controlled coupling reactions, starting from 1,1-difluoro-2,2-dihaloethenes. However, there are only a few examples of the monoarylation of symmetric gem-dihaloethenes [7], because this type of selective reaction requires steric and/or electronic effects of vinylic substituents [8]. Although the monoarylation of symmetric gem-dichloroethenes has been reported, the low reactivities of their chlorine substituents might prevent a second arylation toward diarylated ethenes. Using highly reactive coupling partners of the arylating agents and/or vinyl halides (or pseudohalides) would easily induce double arylation, leading to undesirable symmetrical byproducts. Therefore, the selective synthesis of unsymmetrically disubstituted 1,1-diaryl-2,2-difluoroethenes is a significant challenge.

To solve these synthetic problems, we sought to find an appropriate ligand for each coupling step, preventing the formation of symmetrical 1,1-diaryl-2,2-difluoroethenes 3 . We eventually achieved the selective synthesis of unsymmetrical 1,1-diaryl-2,2-difluoroethenes 4 by choosing ligands to control the reactivities in the first and second arylations of 1,1-dibromo-2,2-difluoroethene (1), which is commercially available (Scheme 2) [9]. 


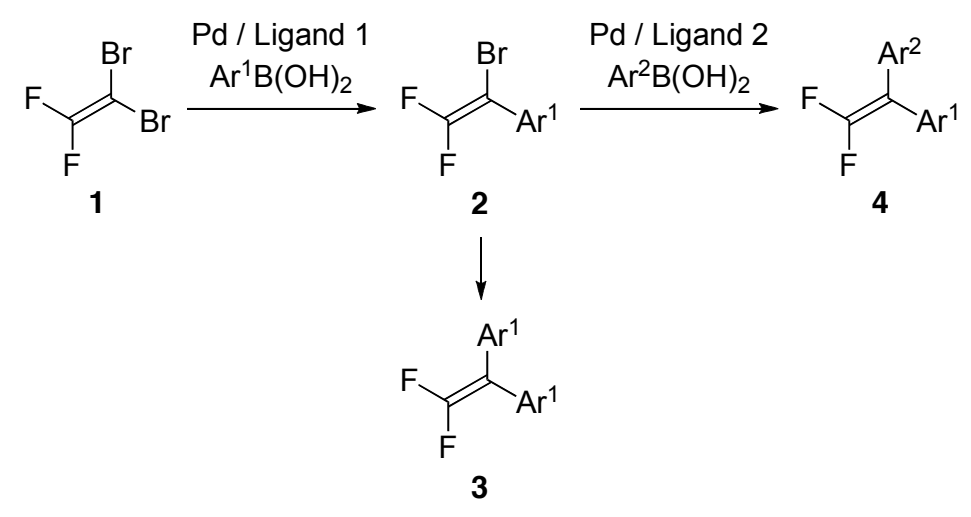

Scheme 2. Strategy for the synthesis of unsymmetrical 1,1-diaryl-2,2-difluoroethenes 4 via stepwise coupling of 1,1-dibromo-2,2-difluoroethene (1).

\section{Results and discussion}

2.1. The first coupling: Selective synthesis of 1-aryl-1-bromo-2,2-difluoroethene 2 from

\section{1,1-dibromo-2,2-difluoroethene (1)}

To synthesize unsymmetrically disubstituted 1,1-diaryl-2,2-difluoroethenes $\mathbf{4}$, we first sought suitable conditions for selective monoarylation in the Suzuki-Miyaura coupling between 1,1-dibromo-2,2-difluoroethene (1) and phenylboronic acid, using cesium fluoride as a base (Table 1). The choice of ligands used with $\mathrm{Pd}_{2} \mathrm{dba}_{3} \cdot \mathrm{CHCl}_{3}(\mathrm{dba}$ = dibenzylideneacetone) was found to be critical for the yield of the monoarylated product, 1-bromo-1-phenyl-2,2-difluoroethene (2a, Entries 1-8). Among the ligands that were screened, 1,1-bis(diphenylphosphino)methane (dppm) gave the highest yield of 2a (Entry 8), suppressing the formation of the diarylated product 3a. Co-solvents used with water (Entries 9-14) at different ratios (Entries 15-16) were subsequently examined. Ether-type solvents typically gave good yield and selectivity of 2a (Entries 9-11), and dioxane gave the highest yield of 2a in a short reaction time (Entry 11). Finally, we found that the optimum dioxane/water ratio was 2:1 (Entry 16), which gave, almost exclusively, bromodifluorostyrene $\mathbf{2 a}$ in $80 \%$ yield.

Table 1. Effects of the ligand and solvent used in the first Suzuki-Miyaura coupling 


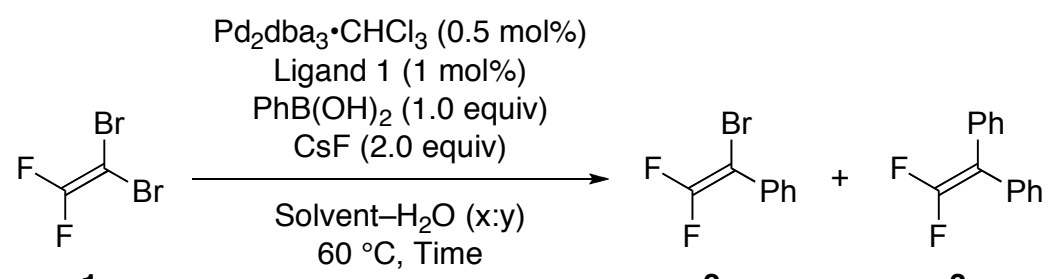

1

$2 a$

$3 a$

\begin{tabular}{|c|c|c|c|c|c|}
\hline Entry & Ligand 1 & Solvent (x:y) & Time / h & $\mathbf{2 a} / \%{ }^{a}$ & $3 a / \% a$ \\
\hline 1 & $\mathrm{PPh}_{3} \mathrm{~b}$ & THF (4:1) & 6 & 51 & Trace \\
\hline 2 & $\mathrm{P}(t-\mathrm{Bu})_{3}{ }^{\mathrm{b}}$ & $\operatorname{THF}(4: 1)$ & 6 & - & - \\
\hline 3 & Cy-JohnPhos ${ }^{b}$ & THF (4:1) & 6 & - & - \\
\hline 4 & $\mathrm{AsPh}_{3}{ }^{\mathrm{b}}$ & $\operatorname{THF}(4: 1)$ & 6 & 24 & Trace \\
\hline 5 & Xantphos & THF (4:1) & 6 & 8 & 12 \\
\hline 6 & dppf & THF $(4: 1)$ & 6 & 40 & 1 \\
\hline 7 & dppb & THF (4:1) & 6 & 14 & Trace \\
\hline 8 & dppm & THF (4:1) & 6 & 54 & Trace \\
\hline 9 & dppm & THF (4:1) & 3 & 36 & 2 \\
\hline 10 & dppm & DME $(4: 1)$ & 3 & 49 & 1 \\
\hline 11 & dppm & Dioxane (4:1) & 3 & 70 & 4 \\
\hline 12 & dppm & DMF $(4: 1)$ & 3 & 10 & 4 \\
\hline 13 & dppm & $\operatorname{MeCN}(4: 1)$ & 3 & 36 & 4 \\
\hline 14 & dppm & Toluene $(4: 1)$ & 3 & 23 & Trace \\
\hline 15 & dppm & Dioxane (8:1) & 3 & 53 & 2 \\
\hline 16 & dppm & Dioxane (2:1) & 3 & 80 & 3 \\
\hline
\end{tabular}

${ }^{\text {a }}{ }^{19} \mathrm{~F}$ NMR yield based on $\mathrm{PhCF}_{3} \cdot{ }^{\mathrm{b}} 2 \mathrm{~mol} \%$.

Once the optimal conditions had been determined, we investigated the scope of the substrate (Table 2). Monoarylation with several para-functionalized ( $\mathrm{Me}, \mathrm{Ph}, \mathrm{OMe}$, and F) phenylboronic acids was successfully achieved, providing bromodifluorostyrenes 2a-2e. To the best of our knowledge, this is the first example of the selective monoarylation of symmetrical gem-dibromoalkenes.

Table 2. Monoarylation of 1,1-dibromo-2,2-difluoroethene (1) 


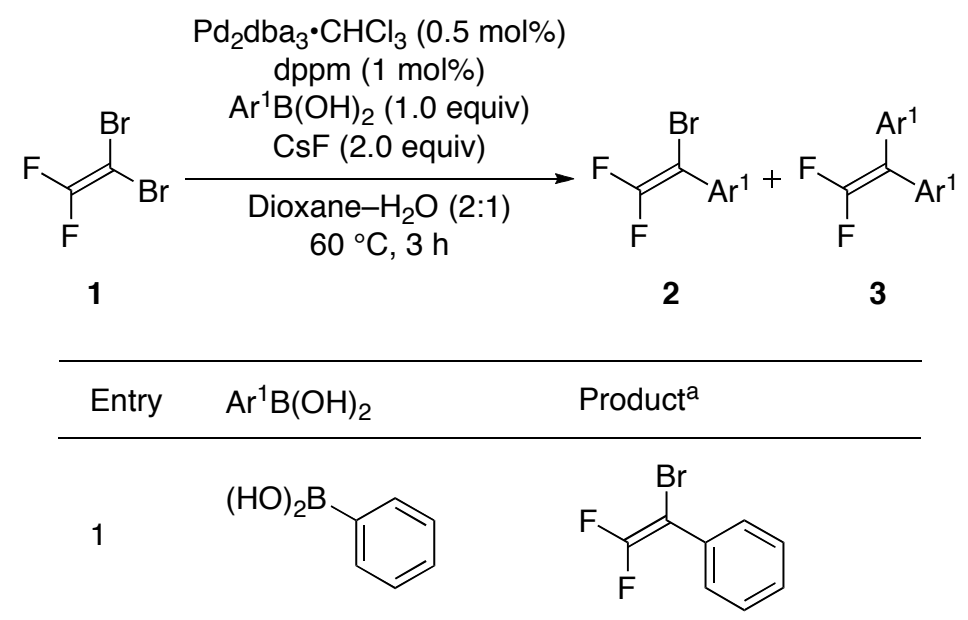

2a, $86 \%$

(3a, 3\%)

2<smiles>Cc1ccc(BOO)cc1</smiles><smiles>Cc1ccc(C(Br)=C(F)F)cc1</smiles>

2b, $69 \%$

(3b, 3\%)

3<smiles>O[BH2-]c1ccc(-c2ccccc2)cc1</smiles><smiles>FC(F)=C(Br)c1ccc(-c2ccccc2)cc1</smiles>

2c, $83 \%$

(3c, 4\%)

4

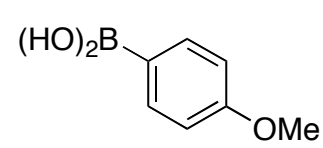<smiles>COc1ccc(C(Br)=C(F)F)cc1</smiles>

2d, $59 \%$

(3d, $5 \%)$

5<smiles>O[B]c1ccc(F)cc1</smiles><smiles>FC(F)=C(Br)c1ccc(F)cc1</smiles>

2e, $68 \%$

(3e, $5 \%)$

a Isolated yield (in parentheses is shown ${ }^{19} \mathrm{~F}$ NMR yield based on $\mathrm{PhCF}_{3}$ ).

2.2. The second coupling: Synthesis of unsymmetrically substituted

\section{1,1-diaryl-2,2-difluoroethenes 4 from 1-aryl-1-bromo-2,2-difluoroethenes 2}

The ligand used for the second coupling in the synthesis of unsymmetrically 
disubstituted 1,1-diaryl-2,2-difluoroethenes 4 was examined next, and the ligands were screened in the Suzuki-Miyaura coupling of monoarylated bromodifluorostyrene 2c with 4-fluorophenylboronic acid, at higher temperatures than in the first step. The second coupling proceeded most effectively using triphenylphosphine, yielding 1,1-diaryl-2,2-difluoroethenes $\mathbf{4 c}$ in $72 \%$ yield (Entry 1). The low yield obtained using dppm indicated that dppm, although highly suitable for the first coupling, effectively suppressed the second coupling (Entry 4).

Table 3. Effects of the ligand in the second Suzuki-Miyaura coupling

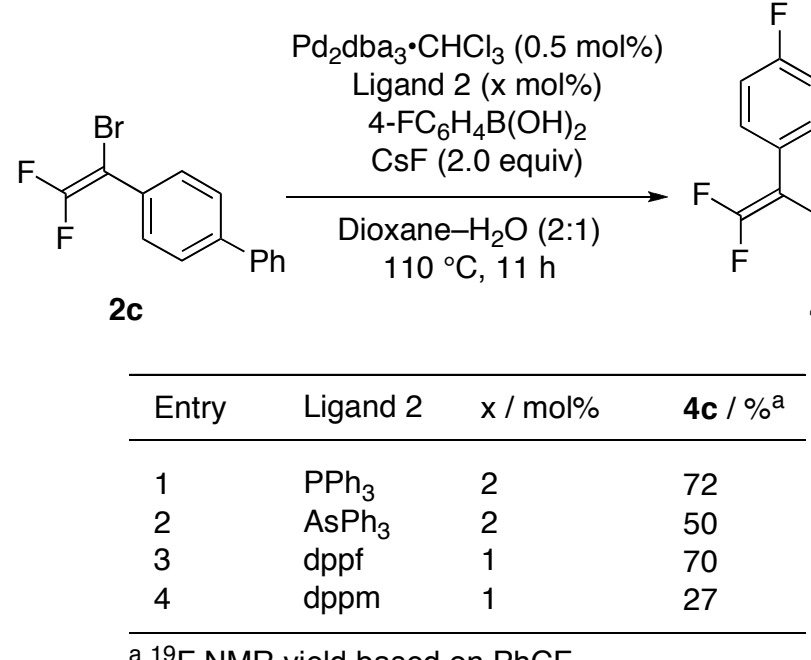

a ${ }^{19} \mathrm{~F}$ NMR yield based on $\mathrm{PhCF}_{3}$.

The synthesis of several unsymmetrically disubstituted 1,1-diaryl-2,2-difluoroethenes 4 was examined under the conditions described above (Table 4). Both para-substituted (Me, OMe, and F; Entries 1-3) and ortho-substituted (Me; Entry 4) phenylboronic acids readily participated in the reaction, affording the desired difluoroethenes $\mathbf{4 a - d}$ in high yields.

Table 4. Synthesis of unsymmetrical 1,1-diaryl-2,2-difluoroethenes 4 


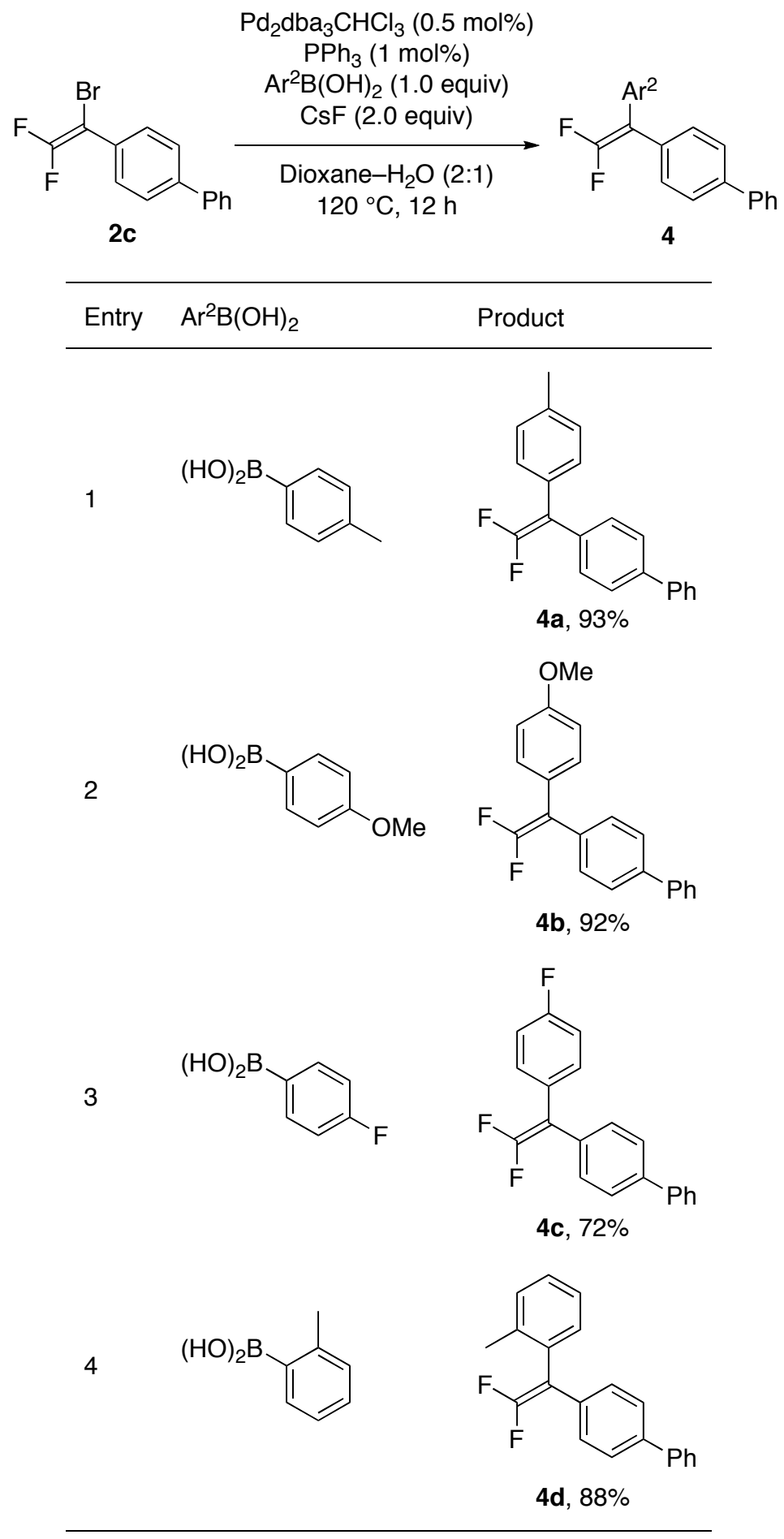

3. Conclusion

We synthesized unsymmetrically disubstituted 1,1-diaryl-2,2-difluoroethenes 4 via stepwise Suzuki-Miyaura coupling reactions, starting from 1,1-dibromo-2,2-difluoroethene (1). The key to success was using the most appropriate

7 
ligand in each coupling step. These protocols provide a versatile method for synthesizing 1,1-diaryl-2,2-difluoroethenes, involving the direct coupling of 1,1-dibromo-2,2-difluoroethene. We expect 1,1-diaryl-2,2-difluoroethenes formed using this method to be biologically active compounds and important intermediates for further chemical transformations.

\section{Experimental}

\subsection{General Information}

IR spectra were recorded on a Horiba FT-300S spectrometer. NMR spectra were recorded on a Bruker Avance 500 spectrometer in $\mathrm{CDCl}_{3}$ at $500 \mathrm{MHz}\left({ }^{1} \mathrm{H} \mathrm{NMR}\right)$, at 126 $\mathrm{MHz}\left({ }^{13} \mathrm{C} \mathrm{NMR}\right)$, and at $470 \mathrm{MHz}\left({ }^{19} \mathrm{~F} \mathrm{NMR}\right)$. Chemical shifts were given in ppm relative to internal $\mathrm{Me}_{4} \mathrm{Si}$ (for ${ }^{1} \mathrm{H} \mathrm{NMR}: \delta=0.00$ ), $\mathrm{CDCl}_{3}$ (for ${ }^{13} \mathrm{C} \mathrm{NMR:} \delta=77.0$ ), and $\mathrm{C}_{6} \mathrm{~F}_{6}$ (for ${ }^{19} \mathrm{~F}$ NMR: $\delta=0.0$ ). High resolution mass spectroscopy (HRMS) was conducted with a JMS-T100GCV spectrometer.

1,1-Dibromo-2,2-difluoroethene, purchased from SynQuest Labs, Inc., was used without further purification. Unless otherwise noted, materials were obtained from commercial sources and used directly without further purification. Column chromatography was performed on silica gel (Kanto, spherical and neutral, 63-210 mesh).

Spectral data for compounds 2a [10,11], 2b [12], 2d [12], and 2e [11] showed good agreement with the literature data. As for compounds $\mathbf{2 a}, \mathbf{2 b}, \mathbf{2 d}$, and $\mathbf{2 e}$, we present only NMR data in this section.

\subsection{Synthesis of 1-aryl-1-bromo-2,2-difluoroethenes 2}

\subsubsection{Typical procedure for the synthesis of 1-aryl-1-bromo-2,2-difluoroethenes 2}

In a pyrex-glass tube were placed phenylboronic acid $(61 \mathrm{mg}, 0.50 \mathrm{mmol}), \mathrm{CsF}(152 \mathrm{mg}$, $1.0 \mathrm{mmol}), \mathrm{Pd}_{2} \mathrm{dba}_{3} \cdot \mathrm{CHCl}_{3}(2.6 \mathrm{mg}, 2.5 \mu \mathrm{mol})$, and dppm (1.9 mg, $\left.4.9 \mu \mathrm{mol}\right)$. After the tube was purged with nitrogen, a pre-degassed mixed solvent $(2.5 \mathrm{~mL}$, 1,4-dioxane/water $=2: 1)$ and 1,1-dibromo-2,2-difluoroethylene $(1,48 \mu \mathrm{L}, 0.50 \mathrm{mmol})$ was added to the tube. After stirring for $3 \mathrm{~h}$ at $60{ }^{\circ} \mathrm{C}$, the mixuture was quenched with $\mathrm{NH}_{4} \mathrm{Cl}$ aq., and organic materials were extracted with ether three times. The combined 
extracts were washed with brine and dried over $\mathrm{Na}_{2} \mathrm{SO}_{4}$. After removal of solvent under reduced pressure, the residue was purified by column chromatography on silica gel (pentane) to give $\mathbf{2 a}(95 \mathrm{mg}, 86 \%)$ as a colorless oil.

\subsubsection{Spectra data of 1-aryl-1-bromo-2,2-difluoroethenes 2}

\subsubsection{1-Bromo-1-phenyl-2,2-difluoroethene (2a)}

Colorless oil. ${ }^{1} \mathrm{H}$ NMR $\left(500 \mathrm{MHz}, \mathrm{CDCl}_{3}\right): \delta 7.33(\mathrm{tt}, J=7.3,1.3 \mathrm{~Hz}, 1 \mathrm{H}), 7.39(\mathrm{dd}, J=$ 7.4, 7.3 Hz, 2H), 7.48-7.51 (m, 2H). $\left.{ }^{13} \mathrm{C} \mathrm{NMR} \mathrm{(126} \mathrm{MHz,} \mathrm{CDCl}_{3}\right): \delta 79.9\left(\mathrm{dd}, J_{\mathrm{CF}}=35\right.$, $26 \mathrm{~Hz}), 128.5,128.80,128.84\left(\mathrm{~d}, J_{\mathrm{CF}}=4 \mathrm{~Hz}\right) 131.6\left(\mathrm{~d}, J_{\mathrm{CF}}=3 \mathrm{~Hz}\right), 153.1\left(\mathrm{dd}, J_{\mathrm{CF}}=295\right.$, $287 \mathrm{~Hz}) .{ }^{19} \mathrm{~F}$ NMR $\left(470 \mathrm{MHz}, \mathrm{CDCl}_{3}\right): \delta 77.1\left(\mathrm{~d}, J_{\mathrm{FF}}=28 \mathrm{~Hz}\right), 83.3\left(\mathrm{~d}, J_{\mathrm{FF}}=28 \mathrm{~Hz}\right)$.

\subsubsection{1-Bromo-1-(4-methylphenyl)-2,2-difluoroethene $(\mathbf{2 b})$}

Colorless oil. ${ }^{1} \mathrm{H}$ NMR $\left(500 \mathrm{MHz}, \mathrm{CDCl}_{3}\right): \delta 2.37$ (s, 3H), $7.19(\mathrm{~d}, J=7.8 \mathrm{~Hz}, 2 \mathrm{H}), 7.37$ $(\mathrm{d}, J=7.8 \mathrm{~Hz}, 2 \mathrm{H}) .{ }^{13} \mathrm{C} \mathrm{NMR}\left(126 \mathrm{MHz}, \mathrm{CDCl}_{3}\right): \delta 21.2,79.9\left(\mathrm{dd}, J_{\mathrm{CF}}=35,26 \mathrm{~Hz}\right)$, $128.7\left(\mathrm{dd}, J_{\mathrm{CF}}=4,4 \mathrm{~Hz}\right), 129.2,130.8\left(\mathrm{dd}, J_{\mathrm{CF}}=4,4 \mathrm{~Hz}\right), 139.0,153.0\left(\mathrm{dd}, J_{\mathrm{CF}}=294\right.$, $286 \mathrm{~Hz}) .{ }^{19} \mathrm{~F} \mathrm{NMR}\left(470 \mathrm{MHz}, \mathrm{CDCl}_{3}\right): \delta 76.5\left(\mathrm{~d}, J_{\mathrm{FF}}=32 \mathrm{~Hz}\right), 82.6\left(\mathrm{~d}, J_{\mathrm{FF}}=32 \mathrm{~Hz}\right)$.

\subsubsection{1-(Biphenyl-4-yl)-1-bromo-2,2-difluoroethene (2c)}

White solid. ${ }^{1} \mathrm{H}$ NMR $\left(500 \mathrm{MHz}, \mathrm{CDCl}_{3}\right): \delta 7.30(\mathrm{tt}, J=7.3,1.3 \mathrm{~Hz}, 1 \mathrm{H}), 7.38(\mathrm{dd}, J=$ 7.7, 7.7 Hz, 2H), 7.49-7.55 (m, 6H). $\left.{ }^{13} \mathrm{C} \mathrm{NMR} \mathrm{(126} \mathrm{MHz,} \mathrm{CDCl}_{3}\right): \delta 79.8\left(\mathrm{dd}, J_{\mathrm{CF}}=34\right.$, $26 \mathrm{~Hz}), 127.1,127.2,127.8,128.9,129.2\left(\mathrm{dd}, J_{\mathrm{CF}}=4,4 \mathrm{~Hz}\right), 130.5\left(\mathrm{~d}, J_{\mathrm{CF}}=4 \mathrm{~Hz}\right)$, $140.1,141.7,153.2\left(\mathrm{dd}, J_{\mathrm{CF}}=295,287 \mathrm{~Hz}\right) .{ }^{19} \mathrm{~F} \mathrm{NMR}\left(470 \mathrm{MHz}, \mathrm{CDCl}_{3}\right): \delta 77.5\left(\mathrm{~d}, J_{\mathrm{FF}}\right.$ $=30 \mathrm{~Hz}), 83.8\left(\mathrm{~d}, J_{\mathrm{FF}}=30 \mathrm{~Hz}\right)$. IR (neat): $\tilde{v}=1709,1290,984,841,764,692 \mathrm{~cm}^{-1}$. HRMS (EI): $m / z$ calcd. for $\mathrm{C}_{14} \mathrm{H}_{9}{ }^{79} \mathrm{BrF}_{2}\left([\mathrm{M}]^{+}\right)$: 293.9856 ; Found: 293.9863 .

\subsubsection{1-Bromo-2,2-difluoro-1-(4-methoxyphenyl)ethene (2d)}

Colorless oil. ${ }^{1} \mathrm{H}$ NMR $\left(500 \mathrm{MHz}, \mathrm{CDCl}_{3}\right): \delta 3.83(\mathrm{~s}, 3 \mathrm{H}), 6.90(\mathrm{~d}, J=9.0 \mathrm{~Hz}, 2 \mathrm{H})$, $7.41(\mathrm{~d}, J=9.0 \mathrm{~Hz}, 2 \mathrm{H}) \cdot{ }^{13} \mathrm{C} \mathrm{NMR}\left(126 \mathrm{MHz}, \mathrm{CDCl}_{3}\right): \delta 55.5,79.6\left(\mathrm{dd}, J_{\mathrm{CF}}=35,26\right.$ 
$\mathrm{Hz}), 113.9,123.8\left(\mathrm{~d}, J_{\mathrm{CF}}=3 \mathrm{~Hz}\right), 130.2\left(\mathrm{dd}, J_{\mathrm{CF}}=3,3 \mathrm{~Hz}\right), 152.8\left(\mathrm{dd}, J_{\mathrm{CF}}=293,286\right.$ $\mathrm{Hz}), 159.9 .{ }^{19} \mathrm{~F} \mathrm{NMR}\left(470 \mathrm{MHz}, \mathrm{CDCl}_{3}\right): \delta 75.7\left(\mathrm{~d}, J_{\mathrm{FF}}=36 \mathrm{~Hz}\right), 81.9\left(\mathrm{~d}, J_{\mathrm{FF}}=36 \mathrm{~Hz}\right)$.

\subsubsection{1-Bromo-2,2-difluoro-1-(4-fluorophenyl)ethene (2e)}

Colorless oil. ${ }^{1} \mathrm{H}$ NMR (500 MHz, $\left.\mathrm{CDCl}_{3}\right): \delta 7.08(\mathrm{dd}, J=8.7,8.7 \mathrm{~Hz}, 2 \mathrm{H}), 7.45-7.49$ $(\mathrm{m}, 2 \mathrm{H}) \cdot{ }^{13} \mathrm{C}$ NMR $\left(126 \mathrm{MHz}, \mathrm{CDCl}_{3}\right): \delta 78.8\left(\mathrm{dd}, J_{\mathrm{CF}}=35,27 \mathrm{~Hz}\right), 115.6\left(\mathrm{~d}, J_{\mathrm{CF}}=22\right.$ $\mathrm{Hz}), 127.7\left(\mathrm{dd}, J_{\mathrm{CF}}=3 \mathrm{~Hz}\right), 130.8(\mathrm{ddd}, J=9,3,3 \mathrm{~Hz}), 153.1\left(\mathrm{dd}, J_{\mathrm{CF}}=294,287 \mathrm{~Hz}\right)$, $162.7\left(\mathrm{~d}, J_{\mathrm{CF}}=250 \mathrm{~Hz}\right) .{ }^{19} \mathrm{~F} \mathrm{NMR}\left(470 \mathrm{MHz}, \mathrm{CDCl}_{3}\right): \delta 49.9-50.0(\mathrm{~m}), 76.9\left(\mathrm{~d}, J_{\mathrm{FF}}=31\right.$ $\mathrm{Hz}), 83.2\left(\mathrm{~d}, J_{\mathrm{FF}}=31 \mathrm{~Hz}\right)$.

\subsection{Synthesis of unsymmetrical 1,1-diaryl-2,2-difluoroethenes 4}

\subsubsection{Typical procedure for the synthesis of 1,1-diaryl-2,2-difluoroethenes 4}

In a pyrex-glass tube were placed 1-bromo-1-(biphenyl-4-yl)-2,2-difluoroethene (2c, 89 mg, $0.30 \mathrm{mmol}$ ), 4-methylphenylboronic acid (45 mg, $0.33 \mathrm{mmol}$ ), CsF (91 mg, 0.60 $\mathrm{mmol}), \mathrm{Pd}_{2} \mathrm{dba}_{3} \cdot \mathrm{CHCl}_{3}(1.6 \mathrm{mg}, 1.5 \mu \mathrm{mol})$, and $\mathrm{PPh}_{3}(1.6 \mathrm{mg}, 6.1 \mu \mathrm{mol})$. After the tube was purged with nitrogen, a pre-degassed mixed solvent $(3.0 \mathrm{~mL}, 1,4$-dioxane/water $=$ 2:1) was added to the tube. After stirring for $12 \mathrm{~h}$ at $120^{\circ} \mathrm{C}$, the mixuture was quenched with $\mathrm{NH}_{4} \mathrm{Cl}$ aq., and organic materials were extracted with ether three times. The combined extracts were washed with brine and dried over $\mathrm{Na}_{2} \mathrm{SO}_{4}$. After removal of solvent under reduced pressure, the residue was purified by column chromatography on silica gel (hexane/EtOAc $=50: 1)$ to give $\mathbf{4 a}(86 \mathrm{mg}, 93 \%)$ as a colorless oil.

\subsubsection{Spectra data of 1,1-diaryl-2,2-difluoroethenes 4}

\subsubsection{1-(Biphenyl-4-yl)-2,2-difluoro-1-(4-methylphenyl)ethene (4a)}

White solid. ${ }^{1} \mathrm{H}$ NMR (500 MHz, $\left.\mathrm{CDCl}_{3}\right): \delta 2.40(\mathrm{~s}, 3 \mathrm{H}), 7.19(\mathrm{~s}, 4 \mathrm{H}), 7.33-7.37(\mathrm{~m}$, $3 \mathrm{H}), 7.44(\mathrm{dd}, J=7.7,7.7 \mathrm{~Hz}, 2 \mathrm{H}), 7.57(\mathrm{~d}, J=8.4 \mathrm{~Hz}, 2 \mathrm{H}), 7.60(\mathrm{~d}, J=7.1 \mathrm{~Hz}, 2 \mathrm{H})$. ${ }^{13} \mathrm{C}$ NMR $\left(126 \mathrm{MHz}, \mathrm{CDCl}_{3}\right): \delta 21.4,96.0\left(\mathrm{dd}, J_{\mathrm{CF}}=18,18 \mathrm{~Hz}\right), 127.20,127.20,127.6$, $129.0,129.3,129.8\left(\mathrm{dd}, J_{\mathrm{CF}}=3,3 \mathrm{~Hz}\right), 130.1\left(\mathrm{dd}, J_{\mathrm{CF}}=3,3 \mathrm{~Hz}\right), 131.4\left(\mathrm{dd}, J_{\mathrm{CF}}=3,3\right.$ 
$\mathrm{Hz}), 133.7\left(\mathrm{dd}, J_{\mathrm{CF}}=4,4 \mathrm{~Hz}\right), 137.6,140.4,140.7,153.9\left(\mathrm{dd}, J_{\mathrm{CF}}=294,294 \mathrm{~Hz}\right) .{ }^{19} \mathrm{~F}$ $\operatorname{NMR}\left(470 \mathrm{MHz}, \mathrm{CDCl}_{3}\right): \delta 73.7\left(\mathrm{~d}, J_{\mathrm{FF}}=33 \mathrm{~Hz}\right), 74.1\left(\mathrm{~d}, J_{\mathrm{FF}}=33 \mathrm{~Hz}\right)$. IR (neat): $\tilde{v}=$ 2924, 1699, 1242, 982, 820, 766, $692 \mathrm{~cm}^{-1}$. HRMS (EI): $\mathrm{m} / z$ calcd. for $\mathrm{C}_{21} \mathrm{H}_{16} \mathrm{~F}_{2}\left([\mathrm{M}]^{+}\right)$: 306.1220; Found: 306.1217.

\subsubsection{1-(Bipnenyl-4-yl)-2,2-difluoro-1-(4-methoxyphenyl)ethene (4b)}

White solid. ${ }^{1} \mathrm{H}$ NMR (500 MHz, $\left.\mathrm{CDCl}_{3}\right): \delta 3.83(\mathrm{~s}, 3 \mathrm{H}), 6.91(\mathrm{~d}, J=9.1 \mathrm{~Hz}, 2 \mathrm{H}), 7.23$ $(\mathrm{d}, J=9.1 \mathrm{~Hz}, 2 \mathrm{H}), 7.33-7.39(\mathrm{~m}, 3 \mathrm{H}), 7.45(\mathrm{dd}, J=7.6,7.6 \mathrm{~Hz}, 2 \mathrm{H}), 7.57(\mathrm{~d}, J=8.4$ $\mathrm{Hz}, 2 \mathrm{H}), 7.60(\mathrm{~d}, J=8.4 \mathrm{~Hz}, 2 \mathrm{H}) \cdot{ }^{13} \mathrm{C} \mathrm{NMR}\left(126 \mathrm{MHz}, \mathrm{CDCl}_{3}\right): \delta 55.4,95.6\left(\mathrm{dd}, J_{\mathrm{CF}}=\right.$ $19,19 \mathrm{~Hz}), 114.0,126.5\left(\mathrm{dd}, J_{\mathrm{CF}}=4,4 \mathrm{~Hz}\right), 127.16,127.16,127.6,128.9,130.0\left(\mathrm{dd}, J_{\mathrm{CF}}\right.$ $=3,3 \mathrm{~Hz}), 131.0\left(\mathrm{dd}, J_{\mathrm{CF}}=3,3 \mathrm{~Hz}\right), 133.7\left(\mathrm{dd}, J_{\mathrm{CF}}=4,4 \mathrm{~Hz}\right), 140.4,140.7,153.8(\mathrm{dd}$, $\left.J_{\mathrm{CF}}=293,293 \mathrm{~Hz}\right), 159.1 .{ }^{19} \mathrm{~F} \mathrm{NMR}\left(470 \mathrm{MHz}, \mathrm{CDCl}_{3}\right): \delta 73.2\left(\mathrm{~d}, J_{\mathrm{FF}}=33 \mathrm{~Hz}\right), 73.6(\mathrm{~d}$, $J_{\mathrm{FF}}=33 \mathrm{~Hz}$ ). IR (neat): $\tilde{v^{2}}=2960,1699,1512,1246,1178,984,835,766 \mathrm{~cm}^{-1}$. HRMS (EI): $m / z$ calcd. for $\mathrm{C}_{21} \mathrm{H}_{16} \mathrm{~F}_{2} \mathrm{O}\left([\mathrm{M}]^{+}\right): 322.1169$; Found: 322.1169 .

\subsubsection{1-(Bipnenyl-4-yl)-2,2-difluoro-1-(4-fluorophenyl)ethene (4c)}

White solid. ${ }^{1} \mathrm{H}$ NMR $\left(500 \mathrm{MHz}, \mathrm{CDCl}_{3}\right): \delta 7.07(\mathrm{dd}, J=8.7,8.7 \mathrm{~Hz}, 2 \mathrm{H}), 7.28$, (dd, $J$ $=8.9,1.0 \mathrm{~Hz}, 2 \mathrm{H}), 7.32(\mathrm{dd}, J=8.5,1.2 \mathrm{~Hz}, 2 \mathrm{H}), 7.36(\mathrm{tt}, J=7.4,1.2 \mathrm{~Hz}, 1 \mathrm{H}), 7.45(\mathrm{dd}$, $J=7.6,7.6 \mathrm{~Hz}, 2 \mathrm{H}), 7.57-7.61(\mathrm{~m}, 4 \mathrm{H}) .{ }^{13} \mathrm{C} \mathrm{NMR}\left(126 \mathrm{MHz}, \mathrm{CDCl}_{3}\right): \delta 95.2\left(\mathrm{dd}, J_{\mathrm{CF}}=\right.$ $19,19 \mathrm{~Hz}), 115.5(\mathrm{~d}, J=22 \mathrm{~Hz}), 127.0,127.1,127.5,128.8,129.8\left(\mathrm{dd}, J_{\mathrm{CF}}=3,3 \mathrm{~Hz}\right)$, $130.1\left(\mathrm{dd}, J_{\mathrm{CF}}=3,3 \mathrm{~Hz}\right), 131.4\left(\mathrm{ddd}, J_{\mathrm{CF}}=8,8,3 \mathrm{~Hz}\right), 133.1\left(\mathrm{dd}, J_{\mathrm{CF}}=3,3 \mathrm{~Hz}\right), 140.4$, $140.5,153.8\left(\mathrm{dd}, J_{\mathrm{CF}}=294,294 \mathrm{~Hz}\right), 162.1\left(\mathrm{~d}, J_{\mathrm{CF}}=248 \mathrm{~Hz}\right) \cdot{ }^{19} \mathrm{~F}$ NMR $(470 \mathrm{MHz}$, $\left.\mathrm{CDCl}_{3}\right): \delta 47.7-47.6(\mathrm{~m}), 74.2\left(\mathrm{~d}, J_{\mathrm{FF}}=32 \mathrm{~Hz}\right), 74.3\left(\mathrm{~d}, J_{\mathrm{FF}}=32 \mathrm{~Hz}\right)$. IR (neat): $\tilde{v}=$ 1707, 1508, 1247, 987, $835 \mathrm{~cm}^{-1}$. HRMS (EI): $\mathrm{m} / z$ calcd. for $\mathrm{C}_{20} \mathrm{H}_{13} \mathrm{~F}_{3}\left([\mathrm{M}]^{+}\right): 310.0969$; Found: 310.0965.

\subsubsection{1-(Bipnenyl-4-yl)-2,2-difluoro-1-(2-methylphenyl)ethene (4d)}

White solid. ${ }^{1} \mathrm{H}$ NMR (500 MHz, $\left.\mathrm{CDCl}_{3}\right): \delta 2.19(\mathrm{~s}, 3 \mathrm{H}), 7.25-7.29(\mathrm{~m}, 6 \mathrm{H}), 7.34(\mathrm{t}, J=$ $7.4 \mathrm{~Hz}, 1 \mathrm{H}), 7.43(\mathrm{dd}, J=7.7,7.7 \mathrm{~Hz}, 2 \mathrm{H}), 7.54(\mathrm{~d}, J=8.4 \mathrm{~Hz}, 2 \mathrm{H}), 7.57$ (d, $J=8.2 \mathrm{~Hz}$, $2 \mathrm{H}) .{ }^{13} \mathrm{C}$ NMR $\left(126 \mathrm{MHz}, \mathrm{CDCl}_{3}\right): \delta 19.6,94.5\left(\mathrm{dd}, J_{\mathrm{CF}}=23,15 \mathrm{~Hz}\right), 126.1,127.0$, 
$127.1,127.4,128.4,128.5\left(\mathrm{dd}, J_{\mathrm{CF}}=6,3 \mathrm{~Hz}\right), 128.8,130.5,131.0\left(\mathrm{dd}, J_{\mathrm{CF}}=2,2 \mathrm{~Hz}\right)$, $132.8\left(\mathrm{~d}, J_{\mathrm{CF}}=4 \mathrm{~Hz}\right), 132.9\left(\mathrm{~d}, J_{\mathrm{CF}}=4 \mathrm{~Hz}\right), 137.7\left(\mathrm{~d}, J_{\mathrm{CF}}=3 \mathrm{~Hz}\right), 139.8,140.5,153.4$ $\left(\mathrm{dd}, J_{\mathrm{CF}}=299,288 \mathrm{~Hz}\right) \cdot{ }^{19} \mathrm{~F} \mathrm{NMR}\left(470 \mathrm{MHz}, \mathrm{CDCl}_{3}\right): \delta 73.1\left(\mathrm{~d}, J_{\mathrm{FF}}=31 \mathrm{~Hz}\right), 78.2(\mathrm{~d}$, $J_{\mathrm{FF}}=31 \mathrm{~Hz}$ ). IR (neat): $\tilde{v}=1705,1487,1244,984,841,764,696 \mathrm{~cm}^{-1}$. HRMS (EI): $m / z$ calcd. for $\mathrm{C}_{21} \mathrm{H}_{16} \mathrm{~F}_{2}\left([\mathrm{M}]^{+}\right)$: 306.1220; Found: 306.1207 .

\section{References and Notes}

[1] N. Hirose, O. Sasaki, Y. Takizawa, Eur. Pat. Appl. 0153145, 1985.

[2] (a) S. Messaoudi, B. Tréguier, A. Hamze, O. Provot, J.-F. Peyrat, J.R. DeLosada, J.-M. Liu, J. Bignon, J. Wdzieczak-Bakala, S. Thoret, J. Dubois, J.-D. Brion, M. Alami, J. Med. Chem. 52 (2009) 4538-4542;

(b) A.L. Ruchelman, H.-W. Man, R. Chen, W. Liu, L. Lu, D. Cedzik, L. Zhang, J. Leisten, A. Collette, R.K. Narla, H.K. Raymon, G.W. Muller, Bioorg. Med. Chem. 19 (2011) 6356-6374.

[3] K.-L. Yu, P. Spinazze, J. Ostrowski, S.J. Currier, E.J. Pack, L. Hammer, T. Roalsvig, J.A. Honeyman, D.R. Tortolani, P.R. Reczek, M.M. Mansuri, J.E. Starret Jr., J. Med. Chem. 39 (1996) 2411-2421;

[4] For recent papers and a review on uses of difluoroalkenes as bioisosteres of ketones, see:

(a) I.A. McDonald, J.M. Lacoste, P. Bey, M.G. Palfreyman, M. Zreika, J. Med. Chem. 28 (1985) 186-193;

(b) M. Bobek, I. Kavai, E. De Clercq, J. Med. Chem. 30 (1987) 1494-1497;

(c) W.R. Moore, G.L. Schatzman, E.T. Jarvi, R.S. Gross, J.R. McCarthy, J. Am. Chem. Soc. 114 (1992) 360-361;

(d) P.M. Weintraub, A.K. Holland, C.A. Gates, W.R. Moore, R.J. Resvick, P. Bey, NP. Peet, Bioorg. Med. Chem. 11 (2003) 427-431;

(e) J.-M. Altenburger, G.Y. Lassalle, M. Matrougui, D. Galtier, J.-C. Jetha, Z. Bocskei, C.N. Berry, C. Lunven, J. Lorrain, J.-P. Herault, P. Schaeffer, S.E. O'Connor, J.-M. Herbert Bioorg. Med. Chem. 12 (2004) 1713-1730;

(f) J. Ichikawa, J. Synth. Org. Chem. Jpn. 68 (2010) 1175-1184.

[5] For synthetic methods for symmetrical 1,1-diaryl-2,2-difluoroethenes, see:

(a) M. Obayashi, E. Ito, K. Matsui, K. Kondo, Tetrahedron Lett. 23 (1982) 2323-2326; 
(b) R. Ocampo, W.R. Dolbier Jr., R. Paredes, J. Fluorine Chem. 88 (1998) 41-50;

(c) G. Mlostoń, J. Romański, H. Heimgartner Heterocycles 50 (1999) 403-410;

(d) I. Nowak, M.J. Robins, Org. Lett. 7 (2005) 721-724;

(e) S.Y. Han, H.Y. Lee, J.H. Jeon, I.H. Jeong, Tetrahedron Lett. 53 (2012) 1833-1836.

[6] For synthetic methods for unsymmetrical 1,1-diaryl-2,2-difluoroethenes, see:

(a) A. Raghavanpillai, D.J. Burton, J. Org. Chem. 71 (2006) 194-201;

(b) J.H. Choi, I.H. Jeong, Tetrahedron Lett. 49 (2008) 952-955;

(c) S.Y. Han, I.H. Jeong, Org. Lett. 12 (2010) 5518-5521.

[7] J. Barluenga, P. Moriel, F. Aznar, C. Valdés, Adv. Synth. Catal. 348 (2006) $347-353$.

[8] (a) Y. Takeda, M. Shimizu, T. Hiyama, Angew. Chem. Int. Ed. 46 (2007) 8659-8661.

(b) D. Alloatti, G. Giannini, W. Cabri, I. Lustrati, M. Marzi, A. Ciacci, G. Gallo, M.O. Tinti, M. Marcellini, T. Riccioni, M.B. Guglielmi, P. Carminati, C. Pisano, J. Med. Chem. 51 (2008) 2708-2721.

(c) G. Chelucci, Chem. Rev. 112 (2012) 1344-1462, and references cited therein.

[9] Although synthesis of unsymmetrical 1,1-diaryl-2,2-difluoroethenes had been reported once by the Suzuki-Miyaura coupling of 1,1-dichloro-2,2-difluoroethene using a water-soluble phosphine ligand, the paper was retracted later, see: J. Pschierer, N. Perschek, H. Plenio, Green Chem. 12 (2010) 636-642. Retraction was endorsed by Sarah Ruthven, Editor, and published on 15th September 2010.

[10] D.J. Burton, A.L. Anderson, R. Takei, HF. Koch, T.L. Shih, J. Fluorine Chem. 16 (1980) 229-235.

[11] R. Anilkumar, D.J. Burton, J. Fluorine Chem. 125 (2004) 561-566.

[12] B.V. Nguyen, D.J. Burton, J. Org. Chem. 63 (1998) 1714-1715. 